\title{
The Road to Approval: a Perspective on the Role of Icosapent Ethyl in Cardiovascular Risk Reduction
}

\author{
Xiaowen Wang $^{1} \cdot$ Subodh Verma ${ }^{2} \cdot$ R. Preston Mason ${ }^{1,3} \cdot$ Deepak L. Bhatt $^{1}$ \\ Accepted: 10 September 2020 / Published online: 23 October 2020 \\ (C) The Author(s) 2020
}

\begin{abstract}
Purpose of Review Epidemiological studies have long suggested the cardiovascular benefits of omega-3 fatty acids (OM3FAs). However, until recently, clinical trials using OM3FAs have been largely negative with respect to their cardioprotective effects. In this review, we aim to summarize key clinical trials, examine the clinical benefits of eicosapentaenoic acid (EPA) and potential mechanisms, and review the changes in guidelines and recommendations.

Recent Findings The Reduction of Cardiovascular Events with Icosapent Ethyl-Intervention Trial (REDUCE-IT) has demonstrated significant cardiovascular mortality benefits of purified EPA ethyl ester, with a $25 \%$ relative risk reduction in major cardiovascular events.

Summary As first of its class to be approved, icosapent ethyl offers a new option to further reduce cardiovascular risks in patients already treated with maximally tolerated statins.
\end{abstract}

Keywords Icosapent ethyl $\cdot$ Eicosapentaenoic acid $\cdot$ Triglyceride $\cdot$ Cholesterol

\section{Introduction}

Despite significant advances in our understanding of lifestyle management and pharmacotherapies, cardiovascular $(\mathrm{CV})$ disease remains a major cause of adult mortality both in the US and worldwide [1]. While the wide use of statins in primary and secondary prevention has significantly improved $\mathrm{CV}$ outcomes, the quest to identify other therapeutic targets to further reduce $\mathrm{CV}$ risk continues [2].

One such therapeutic target is triglycerides. Epidemiological studies suggest that elevated triglyceride levels are associated with increased CV morbidity and mortality, and increased dietary intake of omega-3 fatty acids (OM3FAs) is associated with lower

This article is part of the Topical Collection on Macrovascular Complications in Diabetes

Deepak L. Bhatt

DLBhattmd@post.Harvard.edu

1 Brigham and Women's Hospital and Harvard Medical School, 75 Francis Street, Boston, MA 02115, USA

2 University of Toronto, Toronto, Canada

3 Elucida Research LLC, Beverly, MA, USA
CV mortality [3•, 4-11]. Importantly, this association has been observed at even modest levels of hypertriglyceridemia. Several studies have shown that OM3FAs are effective in lowering triglycerides; however, it had been unclear whether this could translate to any clinical CV benefits [12-14]. In this review, we will summarize key clinical trials that led to the landmark trial REDUCE-IT (Fig. 1), which demonstrated the clinical benefits of icosapent ethyl (Fig. 2) and led to its approval for CV risk reduction (Fig. 3).

\section{Existing Clinical Trials}

Based on epidemiological observations, many prior studies have attempted to examine the clinical effects of lowering triglycerides [3•]. Three major classes of medications have been studied to lower triglycerides or increase HDL: fibrates, niacin, and OM3FAs. While earlier studies showed promising results in using fibrates to improve clinical outcomes before routine moderate and high intensity statin use [15-17], more contemporary trials using fibrates in addition to statins did not demonstrate such clinical benefits [18]. Similarly, studies such as AIM-HIGH and HPS2-THRIVE did not show any clinical benefits of niacin when added to a background of statins [19, 20, 21•]. 


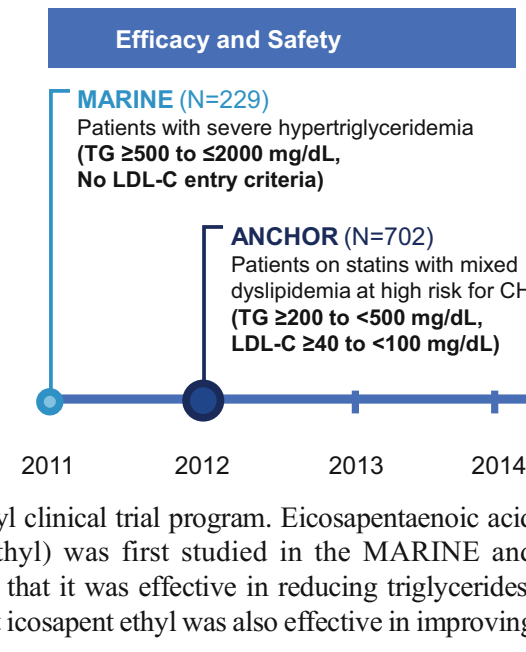

Many recent studies in OM3FAs showed no significant benefits. The Vitamin D and Omega-3 Trial (VITAL) used a two-by-two factorial design to evaluate the effects of vitamin D3 and OM3FAs at a dose of $1 \mathrm{~g}$ per day (containing $380 \mathrm{mg}$ of docosahexaenoic acid [DHA] and $460 \mathrm{mg}$ of eicosapentaenoic acid [EPA]) or placebo in primary prevention of $\mathrm{CV}$ disease and cancer [22 $\bullet$. After randomization of 25,871 participants and a median follow-up of 5.3 years, there was no significant reduction in the rate of major $\mathrm{CV}$ events (hazard ratio [HR] 0.92, 95\% confidence interval [CI] 0.801.06) [22•]. The ASCEND (A Study of CV Events in Diabetes) Study Group evaluated whether OM3FAs improve $\mathrm{CV}$ outcomes in patients with diabetes without known $\mathrm{CV}$ disease [23•]. The investigators randomized 15,480 participants to receive $1 \mathrm{~g}$ of OM3FAs (a mixture of DHA and EPA) or placebo (olive oil) daily. During a mean follow-up
CV Outcomes

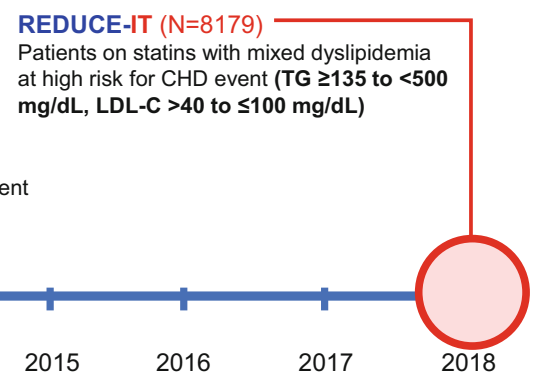

clinical outcomes and reducing cardiovascular mortality. $C H D$ coronary heart disease, $C V$ cardiovascular, $L D L-C$ low-density lipoprotein cholesterol, $T G$ triglycerides

of 7.4 years, there was no significant difference in the rate of first serious vascular events $(8.9 \%$ of patients in the experimental group versus $9.2 \%$ in the placebo group, rate ratio $0.97,95 \%$ CI $0.87-1.08$ ) [23•]. Previously, in patients with acute coronary syndrome, OM3FAs in addition to contemporary guideline-directed therapy (including statins and antiplatelets) did not show a decrease in sudden cardiac death or major CV events in the Alpha Omega and OMEGA trials $[24,25]$.

Two older studies showed clinical benefits of OM3FAs in $\mathrm{CV}$ outcomes. In the open-label GISSI-P study, investigators enrolled 11,324 participants with recent myocardial infarction (MI) and randomized them to vitamin E or OM3FAs using a two-way factorial design [26•]. After 42 months of follow-up, the investigators did not find any clinical effects with vitamin $\mathrm{E}$, but found a lower risk of death, nonfatal MI, and stroke in
Fig. 2 Intestinal processing and absorption of icosapent ethyl (IPE) and its conversion to eicosapentaenoic acid (EPA). $C V$ cardiovascular, FDA Food and Drug Administration

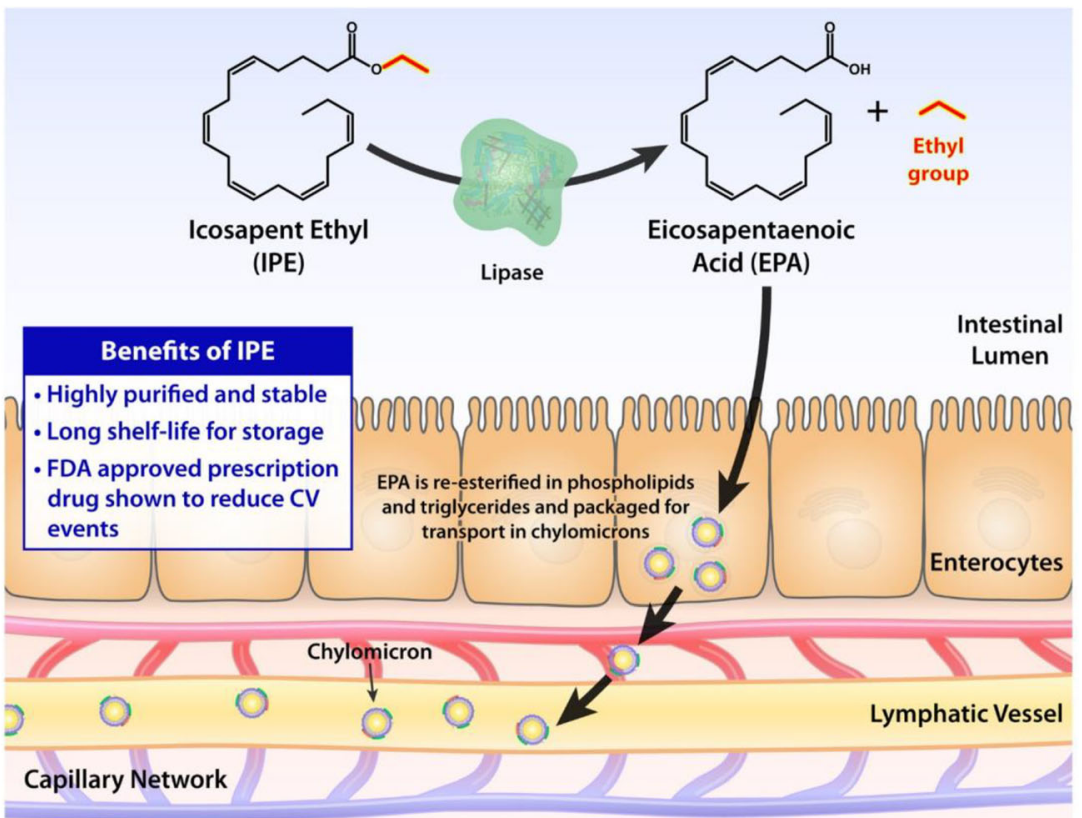


Fig. 3 The road to approval: from MARINE and ANCHOR to REDUCE-IT and US FDA and Health Canada approval. Along the way, there have been several key presentations and publications from REDUCE-IT, as well as numerous guideline endorsements. $A C C$ American College of Cardiology, AHA American Heart Association, $O M 3 F A$ omega-3 fatty acid, $A D A$ American Diabetes Association, EAS European Atherosclerosis Society, ESC European Society of Cardiology, NLA National Lipid Association, EMDAC

Endocrinologic and Metabolic Drugs Advisory Committee, FDA Food and Drug Administration

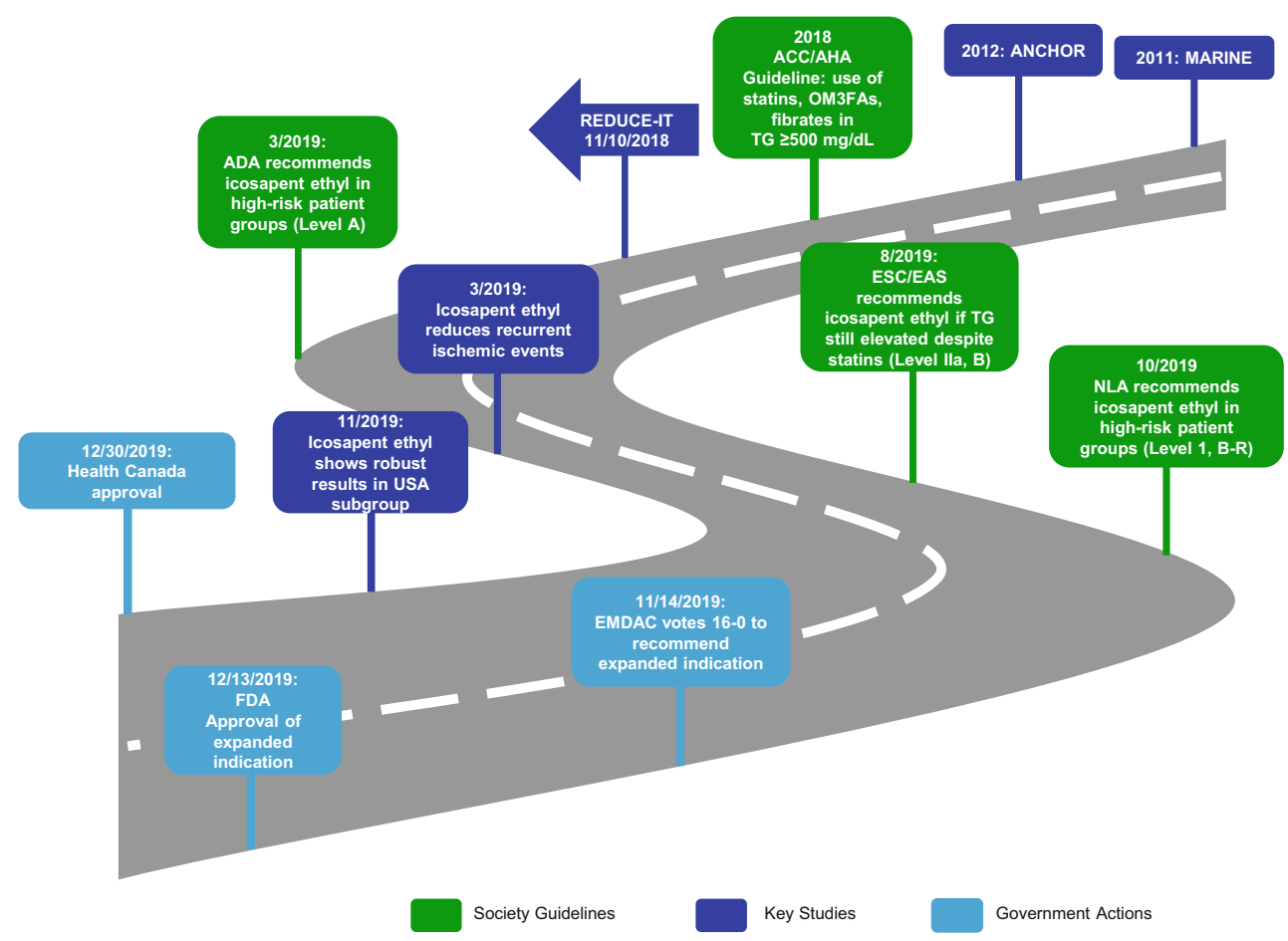

the group that received $n-3$ polyunsaturated fatty acids (PUFAs). Of the 5666 participants who received n-3 PUFAs (including those who received vitamin $\mathrm{E}$ and $\mathrm{n}-3$ PUFAs), 715 met the primary endpoint of death, nonfatal MI, or nonfatal stroke (relative risk [RR] 0.90, 95\% CI 0.82-0.99 for two-way analysis); in the n-3 PUFAs only group (2836 patients), 356 patients met the primary endpoint (RR $0.85,95 \%$ CI 0.74 0.98 for four-way analysis) [26•]. Notably, in the GISSI-P study, only $4.7 \%$ of participants were on cholesterollowering drugs at baseline, which increased to $45.5 \%$ at 42 months [26•]. The Japanese study JELIS was also an open-label study [27•]. However, unlike the GISSI-P study in which a combination of EPA and DHA were used, the JELIS study used a highly purified prescription EPA ethyl ester in addition to statins. In JELIS, 18,645 participants with total cholesterol of $6.5 \mathrm{mmol} / \mathrm{L}(251 \mathrm{mg} / \mathrm{dL})$ or greater were randomized to receive either $1.8 \mathrm{~g}$ of ethyl icosapentate (an EPA ethyl ester) with statin or statin only. The study population included both patients for primary and secondary prevention. After a mean follow-up of 4.6 years, the primary endpoint of major coronary events (sudden cardiac death, fatal and nonfatal MI, unstable angina, angioplasty, stenting, or coronary artery bypass graft [CABG]) occurred in $2.8 \%$ patients in the ethyl icosapentate group versus $3.5 \%$ in the control group (19\% relative risk reduction [RRR], $p=0.011$ ). The effect was consistent in both the secondary prevention subgroup $(8.7 \%$ in the ethyl icosapentate group versus $10.7 \%$ in the control group, 19\% RRR, $p=0.048$ ) and in the primary prevention group $(1.4 \%$ in the EPA group versus $1.7 \%$ in the control group, $18 \% \mathrm{RRR}, p=0.13$ ) [27•].
These earlier studies provided important insights for the design of REDUCE-IT (Reduction of CV Events with Icosapent Ethyl-Intervention Trial). First, the dose of the OM3FAs is important to consider. Many of the earlier trials used lower dose of OM3FAs (1-2 g/day), which may not have been sufficient in a Westernized population in which dietary intake of OM3FAs tends to be low. Second, the composition, purity, and stability of OM3FAs merit consideration. The composition of OM3FAs is important, as EPA and DHA have been shown to have different effects on cell membranes [28]. In MARINE and ANCHOR, icosapent ethyl was shown to be effective in reducing triglycerides (without raising LDL-C); however, these trials were not powered for clinical CV outcomes [12, 14].

In REDUCE-IT, 8179 participants were randomly assigned to receive $4 \mathrm{~g}$ ( $2 \mathrm{~g}$ twice daily with meals) of icosapent ethyl or matching placebo [29*0]. Participants were eligible if they were 45 years of age or older with established $\mathrm{CV}$ disease (secondary prevention cohort) or 50 years of age or older with diabetes and at least one additional risk factor (primary prevention cohort). Patients had a low-density lipoprotein (LDL) cholesterol level of 41 to $100 \mathrm{mg} / \mathrm{dL}$ and a fasting triglyceride level of $150-499 \mathrm{mg} / \mathrm{dL}$. The protocol initially allowed for a $10 \%$ lower triglyceride level $(135 \mathrm{mg} / \mathrm{dL})$, due to the inherent variability in triglyceride levels. However, the lower limit was raised to $200 \mathrm{mg} / \mathrm{dL}$ during the trial to ensure there would be enough participants in this range of triglycerides. The primary endpoint of CV death, nonfatal MI, nonfatal stroke, coronary revascularization, or unstable angina occurred in $17.2 \%$ of 
participants in the icosapent ethyl group versus $22.0 \%$ in the placebo group, a RRR of $25 \%$ (HR $0.75,95 \%$ CI $0.68-0.83$, $p=0.00000001$ ) [29••, 30]. In a subgroup analysis, a similar degree of benefit was seen in patients with diabetes, either with or without baseline CV disease [31].

In a subsequent analysis, icosapent ethyl was shown to not only reduce the first ischemic event, but to also reduce the burden of subsequent and total ischemic events by $30 \%$ (RR $0.70,95 \%$ CI $0.62-0.78, p<0.0001)[30,32 \bullet, 33,34]$. A subsequent analysis showed that participants in the icosapent ethyl group had a significantly lower rate of coronary revascularization - both percutaneous coronary intervention (PCI) and CABG [35]. Studies have shown that REDUCEIT has wide generalizability. Examining an international registry that does not include the US population (which has a high prevalence of hypertriglyceridemia), CLARIFY (ProspeCtive observational LongitudinAl RegIstry oF patients with stable coronary arterY disease) found that $15.5 \%$ of patients who had complete data would have been eligible to enroll in REDUCEIT [36•]. In a Canadian cohort of 196,717 individuals with atherosclerotic CV disease (ASCVD), 25\% were eligible for icosapent ethyl based on their lipid profile (well-controlled LDL with elevated triglycerides in the $135-499 \mathrm{mg} / \mathrm{dL}$ range) [37]. Furthermore, among the 3146 participants in REDUCEIT who were randomized in the US, there was a robust reduction in $\mathrm{CV}$ risks, with the primary composite endpoint of $\mathrm{CV}$ death, nonfatal MI, nonfatal stroke, coronary revascularization, or hospitalization from unstable angina occurring in $18.2 \%$ in the icosapent ethyl group versus $24.7 \%$ in the control group (HR 0.69, 95\% CI 0.59-0.80, $p=0.000001$ ) [38••].

Another recent trial, Outcome Study to Assess Statin Residual Risk Reduction with EpaNova in HiGh CV Risk PatienTs with Hypertriglyceridemia (STRENGTH), also examined the effect of OM3FAs in reducing CV morbidity and mortality [39]. In this randomized, double-blind, placebocontrolled trial, 13,086 participants were randomly assigned to Epanova (550 mg of EPA and $200 \mathrm{mg}$ of DHA per $1 \mathrm{~g}$ capsule) $4 \mathrm{~g}$ once daily or matching placebo, with the primary outcome of time to first event of CV death, MI, stroke, coronary revascularization, or unstable angina [39]. However, at the recommendation of the independent data monitoring committee, the study was discontinued early due to its low likelihood of demonstrating a benefit [40]. This is likely related to the different biological properties of EPA and DHA, as discussed below.

\section{Mechanisms}

While REDUCE-IT identified a promising therapeutic target (high-normal to moderately elevated triglycerides) to further reduce $\mathrm{CV}$ risk, the exact mechanisms remain unclear but appear to be mediated by EPA and its downstream antiinflammatory, antithrombotic, and plaque stabilization effects (Fig. 4). The CV benefits from icosapent ethyl are greater than expected from the modest degree of triglyceride lowering in patients with the range of elevated triglycerides in REDUCEIT, especially in the subgroup of $\sim 10 \%$ of patients enrolled with normal baseline triglyceride levels. EPA has been shown to have pleiotropic effects that contribute to the lowering of $\mathrm{CV}$ risk beyond the effects of triglyceride lowering alone. Clinical studies have shown that treatment with EPA increased fibrous cap thickness, suggesting stabilization of fibroatheroma, and decreased coronary plaque volume [41-44]. In the prespecified interim 9-month analysis of the Effect of Vascepa on Progression of Coronary Atherosclerosis in Persons with Elevated Triglycerides on Statin Therapy (EVAPORATE), icosapent ethyl at $4 \mathrm{~g} /$ day was shown to reduce total plaque volume compared with placebo,
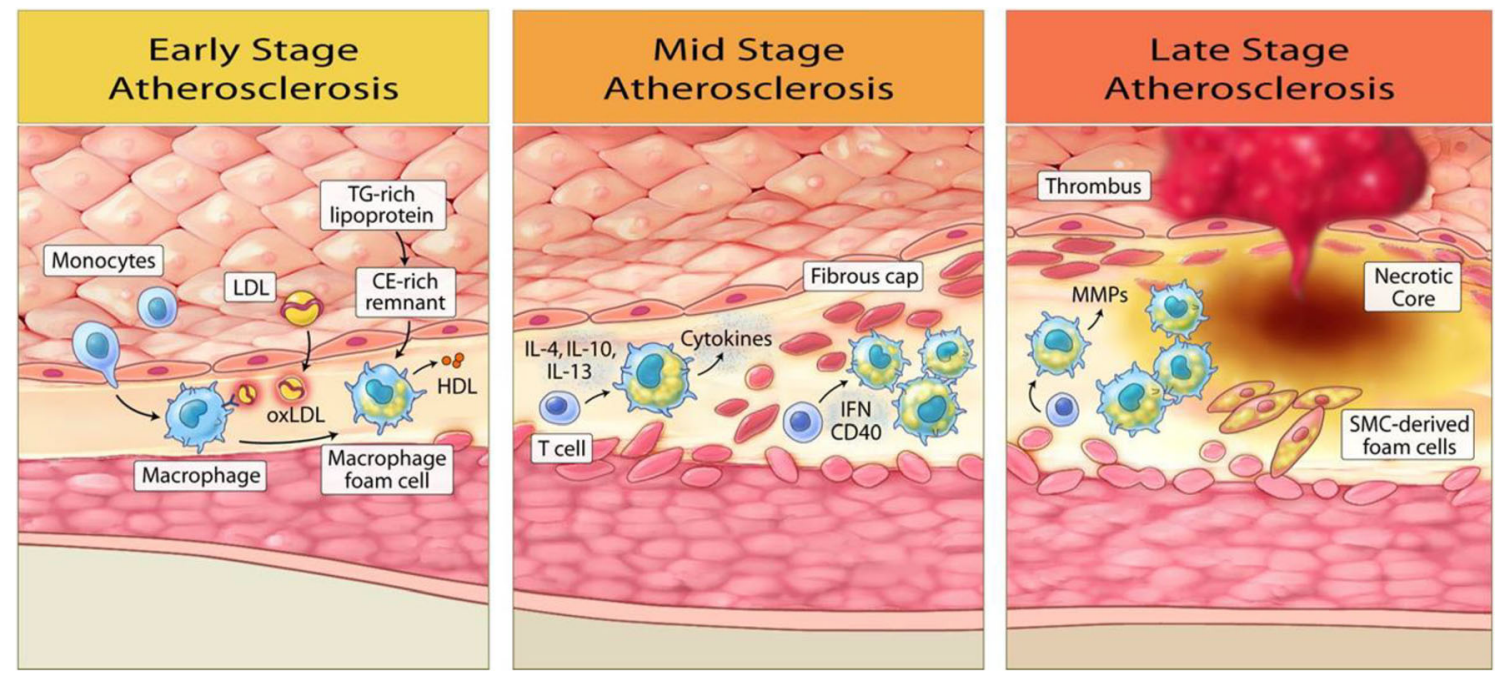

Fig. 4 Icosapent ethyl (IPE) modulates the entire atherosclerosis continuum via eicosapentaenoic acid (EPA) and its downstream anti-inflammatory, antithrombotic, and plaque stabilization effects 
demonstrating a rather early effect [45]. The final 18-month results of EVAPORATE demonstrate significant reduction in several measures of plaque volume and composition [46••]. EPA has also been shown to reduce macrophage accumulation and local inflammatory markers [41, 42]. Inflammation has been increasingly recognized as an underlying driver of atherosclerosis [47, 48]. EPA is involved in pro-resolving signaling pathways that lead to decreased oxidized LDL (oxLDL) uptake, which plays a crucial role in atherosclerosis [49]. EPA's observed effects on endothelial function were also a possible contributor to icosapent ethyl's CV benefits, as indicated by studies demonstrating lower markers of endothelial dysfunction with treatment [50]. In isolated human endothelium, the endothelial benefits of EPA were enhanced when combined with a statin, compared with DHA [28]. In a more recent analysis of REDUCE-IT examining the relationship between EPA level and CV outcomes, patients in the icosapent ethyl group had a higher level of EPA at 1 year and 5 years, and on-treatment EPA level correlated with CV outcomes [51]. Interestingly, the effects of icosapent ethyl were consistent across the full range of baseline triglyceride levels, and the CV benefits were higher than expected for the level of triglyceride reduction, further supporting the notion that the benefits of icosapent ethyl were not simply due to the improvement of other biomarkers.

The differences between EPA and DHA may have contributed to the differences in results between REDUCE-IT and STRENGTH. Studies have shown that EPA and DHA integrate into the cell membrane and interact with cholesterol differently $[52,53 \bullet \cdot$. DHA has been observed to undergo rapid conformational changes in the cell membrane, promoting cholesterol-rich domains that likely precipitate unstable atherosclerotic plaques, while EPA does not. EPA also appears to have antioxidant effects that are not reproduced with DHA, including under conditions of high glucose [53••, 54]. The antioxidant effects of EPA in both membranes and atherogenic lipoproteins were also superior to other long-chain PUFAs, indicating a favorable combination of carbon chain length and degree of unsaturation for EPA [55]. EPA has been observed to reduce inflammatory markers such as highsensitivity C-reactive protein, which was not observed in EPA and DHA mixtures [56, 57, 58・•]. This antiinflammatory effect likely has a large contribution to EPA's $\mathrm{CV}$ benefits beyond the level of triglyceride reduction [58••].

Another hypothesized benefit of icosapent ethyl is its antithrombotic effect. OM3FAs, in particular EPA, can integrate into the platelet membrane and replace omega- 6 fatty acids $[59,60]$. Arachidonic acid (AA), a type of omega-6 fatty acid, plays an integral role in platelet function [59-61]. $\mathrm{AA}$ is oxidized by cyclooxygenase (COX)- 1 to generate prostaglandins and thromboxanes, including thromboxane A2 that facilitates platelet activation $[60,61]$. Instead of generating thromboxane A2, EPA leads to production of thromboxane $\mathrm{A} 3$, which has less platelet activation effect than thromboxane A2 [61]. With increased EPA/AA ratio, functional thromboxane $\mathrm{A} 2$ decreases, thereby exerting an antithrombotic effect $[59,60]$. Indeed, animal models have shown that OM3FAs affect platelet aggregation, thereby reducing thrombus formation $[62,63]$. In combination with a statin, EPA also enhances endothelial release of nitric oxide, a potent platelet inhibitor [28]. Several human studies have also demonstrated the antithrombotic effects of OM3FAs. In one study involving 30 participants who were resistant to lowdose aspirin, participants were randomized to receive fulldose aspirin or to receive OM3FAs with low-dose aspirin. Similar to participants treated with high-dose aspirin, those treated with OM3FAs and low-dose aspirin demonstrated reduced platelet activity [59]. In another study, participants with stable coronary disease undergoing PCI were randomized to receive dual antiplatelet therapy (DAPT) with aspirin and clopidogrel, or DAPT plus $1 \mathrm{~g}$ of OM3FAs; patients who received OM3FAs had better platelet response to clopidogrel [64]. OM3FAs' antithrombotic effects may account for the trend towards higher rates of serious bleeding in the icosapent ethyl group ( $2.7 \%$ vs. $2.1 \%, p=0.06)[29 \bullet \bullet]$.

Adverse effects noted in REDUCE-IT included the elevated risks of hospitalization for atrial fibrillation or atrial flutter in patients treated with icosapent ethyl $(3.1 \%$ in the icosapent ethyl group vs. $2.1 \%$ in the control group, $p=0.004$ ) [29・•]. The underlying mechanism of this observation is unclear. In one animal model, an antiarrhythmic effect was observed with consumption of DHA but not EPA [65]. However, in another animal study, both DHA and EPA were observed to have antiarrhythmic properties, likely due to their effects on sodium currents [66]. Several human studies have attempted to assess the effects of OM3FAs in patients with high risks of arrhythmia, such as patients with implantable cardioverter defibrillators or after cardiac surgery [67-71]. The results of these studies have been mixed, and further research is needed to understand the effects of OM3FAs on atrial and ventricular myocardium [67-71].

\section{Current Guidelines and Future Directions}

The 2018 American College of Cardiology/American Heart Association (ACC/AHA) guidelines on lipids, written prior to the publication of the REDUCE-IT trial results, recognized the elevated ASCVD risks in individuals with hypertriglyceridemia $(\geq 175 \mathrm{mg} / \mathrm{dL}$ ) and recommend the use of statins, OM3FAs, or fibrates in such individuals in addition to lifestyle modification and addressing secondary causes [72]. The 2019 European Society of Cardiology (ESC) guidelines recommend using statins as the drug of first choice in high-risk patients with triglycerides $>200 \mathrm{mg} / \mathrm{dL}$ (class I, level B) [73]. These guidelines have already incorporated the findings of 
REDUCE-IT and recommend the use of icosapent ethyl if triglyceride levels are still elevated despite statin treatment (class IIa, level B) [73]. In the American Diabetes Association (ADA)'s 2020 "Standards of Medical Care in Diabetes," patients who are already on statins with wellcontrolled LDL-C but have triglycerides of $135-499 \mathrm{mg} / \mathrm{dL}$, the addition of icosapent ethyl "can be considered to reduce cardiovascular risk" (level A) [74]. The National Lipid Association (NLA) recommends treatment with icosapent eth$\mathrm{yl}$ in patients "age $\geq 45$ years with clinical ASCVD, or age $\geq$ 50 years with diabetes requiring medication plus $\geq 1$ additional risk factor, with fasting triglyceride 135 to $499 \mathrm{mg} / \mathrm{dL}$ on high-intensity or maximally tolerated statin therapy (土ezetimibe)" (class I, level B-R) [75]. Similarly, the Brazilian Society of Cardiology also updated its recommendations to use icosapent ethyl in high-risk patients already on statins with elevated triglycerides (class I, level B) [76]. An AHA Statement on stable coronary artery disease (CAD) in patients with diabetes stated that "Icosapent ethyl is the first non-LDL-focused lipid therapy to demonstrate cardiovascular benefit and should be considered first-line therapy for patients with [type 2 diabetes mellitus] and CAD whose triglycerides remain elevated $(>135 \mathrm{mg} / \mathrm{dL})$ despite maximally tolerated statin and lifestyle changes $[77 \bullet \bullet] . "$

In addition to its clinical effectiveness, icosapent ethyl was also determined to be cost-effective. The Institute for Clinical and Economic Review (ICER) estimated icosapent ethyl to have an annual wholesale acquisition cost (WAC) of $\$ 3699$ (rebated cost $\sim \$ 1625$ ), lower than ICER's value-based price benchmark of $\$ 6300-\$ 9200$ per year [78]. In a separate costeffectiveness analysis, at a cost of $\$ 4.16$ per day, icosapent ethyl was shown to be a dominant strategy in the majority of scenario analyses, which is not typically seen with branded medications or devices and previously seen with aspirin or generic statins for secondary prevention [79].

In addition to endorsements from different academic societies and guidelines, icosapent ethyl also received endorsement from government regulatory agencies. Previously, icosapent ethyl was approved as an adjunct to diet to lower triglycerides in adult patients with severe hypertriglyceridemia (triglycerides $\geq 500 \mathrm{mg} / \mathrm{dL}$ ) to, in essence, reduce the risk of pancreatitis. On November 14, 2019, the Endocrinologic and Metabolic Drugs Advisory Committee (EMDAC) voted unanimously (16-0) to recommend approval of icosapent ethyl for the indication of reducing CV events [80]. On December 13, 2019, icosapent ethyl was approved by the Food and Drug Administration (FDA) to be used as an add-on to maximally tolerated statin therapy in patients with elevated triglycerides $(\geq 150 \mathrm{mg} / \mathrm{dL}$, with no specification of the need for fasting) with established CV disease or with diabetes plus two or more $\mathrm{CV}$ risk factors [81, 82]. Icosapent ethyl was approved by Health Canada on December 30, 2019. Icosapent ethyl was further endorsed by the US House of Representatives Committee on Appropriations that urged the National Heart, Lung, and Blood Institute (NHLBI) "to support research in this area as well as efforts, particularly through the 'Know Your Numbers' campaign, to promote awareness among physicians and patients of the residual cardiovascular risks beyond statin therapy and the importance of taking preventative action to reduce this risk." [83]

\section{Conclusion}

Despite advances in our understanding of $\mathrm{CV}$ risks and use of high-intensity statins, there is still a significant need to further reduce $\mathrm{CV}$ risks in patients who are already on optimal statin therapy. Icosapent ethyl provides an efficacious and safe option to reduce triglycerides and further reduce $\mathrm{CV}$ mortality and morbidity. While its mechanisms are still to be fully elucidated, the benefits of icosapent ethyl outweigh what would be expected for the degree of triglyceride lowering and are likely attributable to its anti-inflammatory and antithrombotic properties. Icosapent ethyl has been recommended by several professional medical societies. As the first drug of its class to be approved, icosapent ethyl ushers in a new era and provides more options to further reduce residual $\mathrm{CV}$ risk in patients with hypertriglyceridemia and other risk factors.

Acknowledgments Open access for this article was paid for by Amarin Pharma, Inc.

\section{Compliance with Ethical Standards}

Conflict of Interest Deepak Bhatt serves as the Chair and International Principal Investigator for REDUCE-IT, with research funding from Amarin to Brigham and Women's Hospital. Dr. Bhatt discloses the following relationships: Advisory Board: Cardax, CellProthera, Cereno Scientific, Elsevier Practice Update Cardiology, Level Ex, Medscape Cardiology, PhaseBio, PLx Pharma, Regado Biosciences; Board of Directors: Boston VA Research Institute, Society of Cardiovascular Patient Care, TobeSoft; Chair: American Heart Association Quality Oversight Committee; Data Monitoring Committees: Baim Institute for Clinical Research (formerly Harvard Clinical Research Institute, for the PORTICO trial, funded by St. Jude Medical, now Abbott), Cleveland Clinic (including for the ExCEED trial, funded by Edwards), Contego Medical (Chair, PERFORMANCE 2), Duke Clinical Research Institute, Mayo Clinic, Mount Sinai School of Medicine (for the ENVISAGE trial, funded by Daiichi Sankyo), Population Health Research Institute; Honoraria: American College of Cardiology (Senior Associate Editor, Clinical Trials and News, ACC.org; Vice Chair, ACC Accreditation Committee), Baim Institute for Clinical Research (formerly Harvard Clinical Research Institute; RE-DUAL PCI clinical trial steering committee funded by Boehringer Ingelheim; AEGIS-II executive committee funded by CSL Behring), Belvoir Publications (Editor in Chief, Harvard Heart Letter), Duke Clinical Research Institute (clinical trial steering committees, including for the PRONOUNCE trial, funded by Ferring Pharmaceuticals), HMP Global (Editor in Chief, Journal of Invasive Cardiology), Journal of the American College of Cardiology (Guest Editor; Associate Editor), K2P (Co-Chair, interdisciplinary curriculum), Level Ex, Medtelligence/ReachMD (CME steering committees), 
MJH Life Sciences, Population Health Research Institute (for the COMPASS operations committee, publications committee, steering committee, and USA national co-leader, funded by Bayer), Slack Publications (Chief Medical Editor, Cardiology Today's Intervention), Society of Cardiovascular Patient Care (Secretary/Treasurer), WebMD (CME steering committees); Other: Clinical Cardiology (Deputy Editor), NCDR-ACTION Registry Steering Committee (Chair), VA CART Research and Publications Committee (Chair); Research Funding: Abbott, Afimmune, Amarin, Amgen, AstraZeneca, Bayer, Boehringer Ingelheim, Bristol-Myers Squibb, Cardax, Chiesi, CSL Behring, Eisai, Ethicon, Ferring Pharmaceuticals, Forest Laboratories, Fractyl, Idorsia, Ironwood, Ischemix, Lexicon, Lilly, Medtronic, Pfizer, PhaseBio, PLx Pharma, Regeneron, Roche, Sanofi Aventis, Synaptic, The Medicines Company; Royalties: Elsevier (Editor, Cardiovascular Intervention: A Companion to Braunwald's Heart Disease); Site Co-Investigator: Biotronik, Boston Scientific, CSI, St. Jude Medical (now Abbott), Svelte; Trustee: American College of Cardiology; unfunded research: FlowCo, Merck, Novo Nordisk, Takeda.

Subodh Verma holds the Canada Research Chair in Cardiovascular Surgery. He has received speaking honoraria and/or grant support from Abbott, AstraZeneca, Bayer, Boehringer-Ingelheim, Janssen, Novonordisk, Novartis, Sun Pharma, Toronto Knowledge Translation Research Group, EOCI Pharmacomm, Merck, LivaNova, Amarin, and HLS Therapeutics. He is President of the Canadian Medical and Surgical Knowledge Translation Research Group.

R. Preston Mason has received consulting and research grants from Amarin Pharma Inc., Novartis, and Pfizer. Advisory Board: Cardax.

Xiaowen Wang declares no potential conflicts of interest.

Human and Animal Rights and Informed Consent This article does not contain any studies with human or animal subjects performed by any of the authors.

Open Access This article is licensed under a Creative Commons Attribution 4.0 International License, which permits use, sharing, adaptation, distribution and reproduction in any medium or format, as long as you give appropriate credit to the original author(s) and the source, provide a link to the Creative Commons licence, and indicate if changes were made. The images or other third party material in this article are included in the article's Creative Commons licence, unless indicated otherwise in a credit line to the material. If material is not included in the article's Creative Commons licence and your intended use is not permitted by statutory regulation or exceeds the permitted use, you will need to obtain permission directly from the copyright holder. To view a copy of this licence, visit http://creativecommons.org/licenses/by/4.0/.

\section{References}

Papers of particular interest, published recently, have been highlighted as:

- Of importance

•- Of major importance

1. Roth GA, Forouzanfar MH, Moran AE, Barber R, Nguyen G, Feigin VL, et al. Demographic and epidemiologic drivers of global cardiovascular mortality. N Engl J Med. 2015;372(14):1333-41. https://doi.org/10.1056/NEJMoa1406656.

2. Xiao C, Dash S, Morgantini C, Hegele RA, Lewis GF. Pharmacological targeting of the atherogenic dyslipidemia complex: the next frontier in CVD prevention beyond lowering LDL cholesterol. Diabetes. 2016;65(7):1767-78. https://doi.org/10. 2337/db16-0046.

3. Ganda OP, Bhatt DL, Mason RP, Miller M, Boden WE. Unmet need for adjunctive dyslipidemia therapy in hypertriglyceridemia management. J Am Coll Cardiol. 2018;72(3):330-43. https://doi. org/10.1016/j.jacc.2018.04.061. A review article that summarized the need to target hypertriglyceridemia to further reduce $\mathrm{CV}$ risk in high-risk individuals. It also summarized several ongoing trials at the time of the review, some of which have reported since then.

4. $\mathrm{Hu} \mathrm{FB}$, Cho E, Rexrode KM, Albert CM, Manson JE. Fish and long-chain omega-3 fatty acid intake and risk of coronary heart disease and total mortality in diabetic women. Circulation. 2003;107(14):1852-7. https://doi.org/10.1161/01.cir.0000062644. 42133.5f.

5. He K, Song Y, Daviglus ML, Liu K, Van Horn L, Dyer AR, et al. Accumulated evidence on fish consumption and coronary heart disease mortality: a meta-analysis of cohort studies. Circulation. 2004;109(22):2705-11. https://doi.org/10.1161/01.cir. $0000132503.19410 .6 \mathrm{~b}$.

6. Kromhout D, Bosschieter EB, de Lezenne Coulander C. The inverse relation between fish consumption and 20-year mortality from coronary heart disease. N Engl J Med. 1985;312(19):1205-9. https://doi.org/10.1056/nejm198505093121901.

7. Siscovick DS, Raghunathan TE, King I, Weinmann S, Wicklund $\mathrm{KG}$, Albright J, et al. Dietary intake and cell membrane levels of long-chain n-3 polyunsaturated fatty acids and the risk of primary cardiac arrest. JAMA. 1995;274(17):1363-7. https://doi.org/10. 1001/jama.1995.03530170043030.

8. Daviglus ML, Stamler J, Orencia AJ, Dyer AR, Liu K, Greenland $\mathrm{P}$, et al. Fish consumption and the 30-year risk of fatal myocardial infarction. N Engl J Med. 1997;336(15):1046-53. https://doi.org/ 10.1056/nejm199704103361502.

9. Albert CM, Campos H, Stampfer MJ, Ridker PM, Manson JE, Willett WC, et al. Blood levels of long-chain n-3 fatty acids and the risk of sudden death. N Engl J Med. 2002;346(15):1113-8. https://doi.org/10.1056/NEJMoa012918.

10. Hu FB, Bronner L, Willett WC, Stampfer MJ, Rexrode KM, Albert $\mathrm{CM}$, et al. Fish and omega-3 fatty acid intake and risk of coronary heart disease in women. JAMA. 2002;287(14):1815-21. https:// doi.org/10.1001/jama.287.14.1815.

11. Albert CM, Hennekens CH, O'Donnell CJ, Ajani UA, Carey VJ, Willett WC, et al. Fish consumption and risk of sudden cardiac death. JAMA. 1998;279(1):23-8. https://doi.org/10.1001/jama. 279.1.23.

12. Bays HE, Ballantyne CM, Kastelein JJ, Isaacsohn JL, Braeckman RA, Soni PN. Eicosapentaenoic acid ethyl ester (AMR101) therapy in patients with very high triglyceride levels (from the multi-center, placebo-controlled, randomized, double-blind, 12-week study with an open-label extension [MARINE] trial). Am J Cardiol. 2011;108(5):682-90. https://doi.org/10.1016/j.amjcard.2011.04. 015.

13. Kastelein JJ, Maki KC, Susekov A, Ezhov M, Nordestgaard BG, Machielse BN, et al. Omega-3 free fatty acids for the treatment of severe hypertriglyceridemia: the epanova for lowering very high triglycerides (EVOLVE) trial. J Clin Lipidol. 2014;8(1):94-106. https://doi.org/10.1016/j.jacl.2013.10.003.

14. Ballantyne CM, Bays HE, Kastelein JJ, Stein E, Isaacsohn JL, Braeckman RA, et al. Efficacy and safety of eicosapentaenoic acid ethyl ester (AMR101) therapy in statin-treated patients with persistent high triglycerides (from the ANCHOR study). Am J Cardiol. 2012;110(7):984-92. https://doi.org/10.1016/j.amjcard.2012.05. 031.

15. Frick MH, Elo O, Haapa K, Heinonen OP, Heinsalmi P, Helo P, et al. Helsinki Heart Study: primary-prevention trial with gemfibrozil in middle-aged men with dyslipidemia. N Engl J Med. 
1987;317(20):1237-45. https://doi.org/10.1056/ nejm198711123172001.

16. The Bezafibrate Infarction Prevention (BIP) Study Group. Secondary prevention by raising HDL cholesterol and reducing triglycerides in patients with coronary artery disease. Circulation. 2000;102(1):21-7. https://doi.org/10.1161/01.cir.102.1.21.

17. Rubins HB, Robins SJ, Collins D, Fye CL, Anderson JW, Elam $\mathrm{MB}$, et al. Gemfibrozil for the secondary prevention of coronary heart disease in men with low levels of high-density lipoprotein cholesterol. N Engl J Med. 1999;341(6):410-8. https://doi.org/10. 1056/nejm199908053410604.

18. Ginsberg HN, Elam MB, Lovato LC, Crouse JR 3rd, Leiter LA, Linz P, et al. Effects of combination lipid therapy in type 2 diabetes mellitus. N Engl J Med. 2010;362(17):1563-74. https://doi.org/10. 1056/NEJMoa1001282.

19. Boden WE, Probstfield JL, Anderson T, Chaitman BR, DesvignesNickens P, Koprowicz K, et al. Niacin in patients with low HDL cholesterol levels receiving intensive statin therapy. N Engl J Med. 2011;365(24):2255-67. https://doi.org/10.1056/NEJMoa1107579.

20. Landray MJ, Haynes R, Hopewell JC, Parish S, Aung T, Tomson J, et al. Effects of extended-release niacin with laropiprant in high-risk patients. N Engl J Med. 2014;371(3):203-12. https://doi.org/10. 1056/NEJMoa1300955.

21. Patel PN, Patel SM, Bhatt DL. Cardiovascular risk reduction with icosapent ethyl. Curr Opin Cardiol. 2019;34(6):721-7. https://doi. org $/ 10.1097 /$ hco.0000000000000678. A review that looked at options to further reduce $\mathrm{CV}$ risk in addition to high-intensity statins, such as fibrates, niacin, and omega-3 fatty acids. This article summarized key triglyceride-lowering trials and compared their outcomes.

22. Manson JE, Cook NR, Lee IM, Christen W, Bassuk SS, Mora S, et al. Marine n-3 fatty acids and prevention of cardiovascular disease and cancer. N Engl J Med. 2018;380(1):23-32. https://doi.org/ 10.1056/NEJMoa1811403. In this randomized, placebocontrolled primary prevention trial, 25,781 participants were randomized to receive vitamin D3, marine $n-3$ fatty acids, or placebo in a two-by-two factorial design. The investigators did not find a significant difference in rates of cancer or major $\mathrm{CV}$ events in participants taking $n-3$ fatty acids.

23. The ASCEND Study Collaborative Group. Effects of n-3 fatty acid supplements in diabetes mellitus. N Engl J Med. 2018;379(16): 1540-50. https://doi.org/10.1056/NEJMoa1804989. A randomized, placebo-controlled trial inolving 15,480 participants with diabetes without established atherosclerosis. The patients were randomized to receive $1 \mathrm{~g}$ of $\mathrm{n}-3$ fatty acids or placebo. There was no difference in first serious $\mathrm{CV}$ events between the two groups after a mean follow-up of 7.4 years.

24. Kromhout D, Giltay EJ, Geleijnse JM. N-3 fatty acids and cardiovascular events after myocardial infarction. N Engl J Med. 2010;363(21):2015-26. https://doi.org/10.1056/NEJMoa1003603.

25. Rauch B, Schiele R, Schneider S, Diller F, Victor N, Gohlke H, et al. OMEGA, a randomized, placebo-controlled trial to test the effect of highly purified omega- 3 fatty acids on top of modern guideline-adjusted therapy after myocardial infarction. Circulation. 2010;122(21):2152-9. https://doi.org/10.1161/ circulationaha.110.948562.

26. GISSI-Prevenzione Investigators. Dietary supplementation with n3 polyunsaturated fatty acids and vitamin e after myocardial infarction: Results of the gissi-prevenzione trial. Lancet. 1999;354(9177): 447-55. In this earlier Italian study (in the 1990's), investigators randomzed 11,324 participants after recent myocardial infarction to receive $\mathrm{n}-3$ fatty acids, vitamin $\mathrm{E}$, both, or placebo. They found a $10-20 \%$ reduction in major $\mathrm{CV}$ events and death in patients who received $\mathbf{n}-3$ fatty acids.

27. Yokoyama M, Origasa H, Matsuzaki M, Matsuzawa Y, Saito Y, Ishikawa $\mathrm{Y}$, et al. Effects of eicosapentaenoic acid on major coronary events in hypercholesterolaemic patients (JELIS): a randomised open-label, blinded endpoint analysis. Lancet. 2007;369(9567):1090-8. https://doi.org/10.1016/s0140-6736(07) 60527-3. In this Japanese study, 18,645 participants were randomized to receive $1.8 \mathrm{~g}$ of $\mathrm{EPA}$ with statin or statin alone. After a mean follow-up of 4.6 years, there was a $19 \%$ relative reduction in major coronary events in patients who received EPA. This was one of the first trials using purified EPA as opposed to a mixture of EPA and DHA.

28. Mason RP, Dawoud H, Jacob RF, Sherratt SCR, Malinski T. Eicosapentaenoic acid improves endothelial function and nitric oxide bioavailability in a manner that is enhanced in combination with a statin. Biomed Pharmacother. 2018;103:1231-7. https://doi.org/ 10.1016/j.biopha.2018.04.118.

29.• Bhatt DL, Steg PG, Miller M, Brinton EA, Jacobson TA, Ketchum $\mathrm{SB}$, et al. Cardiovascular risk reduction with icosapent ethyl for hypertriglyceridemia. N Engl J Med. 2019;380(1):11-22. https:// doi.org/10.1056/NEJMoa1812792. This article reports the results of REDUCE-IT. In this randomized, double-blind, placebo-controlled trial, 8,179 participants with $\mathrm{CV}$ disease or with diabetes and other risk factors and already on statin therapy were randomized to icosapent ethyl ( $2 \mathrm{~g}$ twice daily) or placebo. After a mean follow-up of 4.9 years, there was a $25 \%$ RRR in the composite of $\mathrm{CV}$ death, nonfatal MI, nonfatal stroke, $\mathrm{CV}$ revascularization, or unstable angina.

30. Bhatt DL. REDUCE-IT: residual cardiovascular risk in statintreated patients with elevated triglycerides: now we can REDUCE-IT! Eur Heart J. 2019;40(15):1174-5. https://doi.org/ 10.1093/eurheartj/ehz179.

31. Bhatt DL. Substantial cardiovascular benefit from icosapent ethyl in patients with diabetes: REDUCE-IT DIABETES. American Diabetes Association Virtual Scientific Session. 2020.

32•. Bhatt DL, Steg PG, Miller M, Brinton EA, Jacobson TA, Ketchum $\mathrm{SB}$, et al. Effects of icosapent ethyl on total ischemic events: from REDUCE-IT. J Am Coll Cardiol. 2019;73(22):2791-802. https:// doi.org/10.1016/j.jacc.2019.02.032. In this study, the REDUCEIT investigators used pre-specified statistical methods to examine the differences in subsequent and total ischemic events in patients receiving icosapent ethyl versus placebo. They found a $32 \%$ reduction in first, subsequent, and total ischemic events in the icosapent ethyl group.

33. Boden WE, Bhatt DL, Toth PP, Ray KK, Chapman MJ, Luscher TF. Profound reductions in first and total cardiovascular events with icosapent ethyl in the REDUCE-IT trial: why these results usher in a new era in dyslipidaemia therapeutics. Eur Heart J. 2019;41: 2304-12. https://doi.org/10.1093/eurheartj/ehz778.

34. Bhatt DL, Steg PG, Miller M, Brinton EA, Jacobson TA, Jiao L, et al. Reduction in first and total ischemic events with icosapent ethyl across baseline triglyceride tertiles. J Am Coll Cardiol. 2019;74(8):1159-61. https://doi.org/10.1016/j.jacc.2019.06.043.

35. Peterson BE. Reduction of revascularization in patients with hypertriglyceridemia with icosapent ethyl: insights from REDUCE-IT REVASC. Society of Cardiovascular Angiography and Interventions Virutal Scientific Session. 2020.

36. Picard F, Bhatt DL, Ducrocq G, Elbez Y, Ferrari R, Ford I, et al. Generalizability of the reduce-it trial in patients with stable coronary artery disease. J Am Coll Cardiol. 2019;73(11):1362. https:// doi.org/10.1016/j.jacc.2019.01.016. In this study, the authors evaluated an international cohort of patients with stable CAD (CLARIFY). They analyzed 24,146 patients and found that 15. $5 \%$ of patients met REDUCE-IT inclusion criteria. They extrapolated these data globally and estimated that approximately 17.1 million patients with stable CAD would benefit from icosapent ethyl based on REDUCE-IT inclusion criteria.

37. Lawler PR, Kotrri G, Koh M, Goodman SG, Farkouh ME, Lee DS, et al. Real-world risk of cardiovascular outcomes associated with 
hypertriglyceridaemia among individuals with atherosclerotic cardiovascular disease and potential eligibility for emerging therapies. Eur Heart J. 2019;41(1):86-94. https://doi.org/10.1093/eurheartj/ ehz767.

38.• Bhatt DL, Miller M, Brinton Eliot A, Jacobson Terry A, Steg Ph G, Ketchum Steven B, et al. REDUCE-IT USA: results from the 3146 patients randomized in the United States. Circulation. 2020;141(5): 367-75. https://doi.org/10.1161/CIRCULATIONAHA.119. 044440. In this prespecified subgroup analysis of REDUCEIT, the authors analyzed the 3,146 U.S. participants (out of 8,179 total participants) and found that the U.S. subgroup had a significant reduction in the primary endpoint, with a RRR of $26 \%$, as well as a significant $30 \%$ reduction in mortality.

39. Nicholls SJ, Lincoff AM, Bash D, Ballantyne CM, Barter PJ, Davidson $\mathrm{MH}$, et al. Assessment of omega-3 carboxylic acids in statin-treated patients with high levels of triglycerides and low levels of high-density lipoprotein cholesterol: rationale and design of the strength trial. Clin Cardiol. 2018;41(10):1281-8. https://doi. org/10.1002/clc.23055.

40. AstraZeneca. Update on phase III STRENGTH trial for Epanova in mixed dyslipidaemia. 2020. https://www.astrazeneca.com/mediacentre/press-releases/2020/update-on-phase-iii-strength-trial-forepanova-in-mixed-dyslipidaemia-13012020.html. Accessed 29 March 2020

41. Nishio R, Shinke T, Otake H, Nakagawa M, Nagoshi R, Inoue T, et al. Stabilizing effect of combined eicosapentaenoic acid and statin therapy on coronary thin-cap fibroatheroma. Atherosclerosis. 2014;234(1):114-9. https://doi.org/10.1016/j.atherosclerosis.2014. 02.025 .

42. Yamano T, Kubo T, Shiono Y, Shimamura K, Orii M, Tanimoto T, et al. Impact of eicosapentaenoic acid treatment on the fibrous cap thickness in patients with coronary atherosclerotic plaque: an optical coherence tomography study. J Atheroscler Thromb. 2015;22(1):52-61. https://doi.org/10.5551/jat.25593.

43. Watanabe T, Ando K, Daidoji H, Otaki Y, Sugawara S, Matsui M, et al. A randomized controlled trial of eicosapentaenoic acid in patients with coronary heart disease on statins. J Cardiol. 2017;70(6):537-44. https://doi.org/10.1016/j.jjcc.2017.07.007.

44. Niki T, Wakatsuki T, Yamaguchi K, Taketani Y, Oeduka H, Kusunose K, et al. Effects of the addition of eicosapentaenoic acid to strong statin therapy on inflammatory cytokines and coronary plaque components assessed by integrated backscatter intravascular ultrasound. Circ J. 2016;80(2):450-60. https://doi.org/10.1253/ circj.CJ-15-0813.

45. Budoff MJ, Muhlestein JB, Bhatt DL, Le VT, May HT, Shaikh K, et al. Effect of icosapent ethyl on progression of coronary atherosclerosis in patients with elevated triglycerides on statin therapy: a prospective, placebo-controlled randomized trial (EVAPORATE): interim results. Cardiovasc Res. 2020. https://doi.org/10.1093/cvr/ cvaa184.

46.• Budoff MJ, Bhatt DL, Kinninger A, Lakshmanan S, Muhlestein JB, Le VT, et al. Effect of icosapent ethyl on progression of coronary atherosclerosis in patients with elevated triglycerides on statin therapy: final results of the EVAPORATE trial. Eur Heart J. 2020. https://doi.org/10.1093/eurheartj/ehaa652. In this study, the 18month results of the EVAPORATE trial showed significant differences in several measures of plaque volume and composition with icosapent ethyl versus placebo, suggesting a potential cardioprotective mechanism of icosapent ethyl by plaque modification.

47. Lawler PR, Bhatt DL, Godoy LC, Lüscher TF, Bonow RO, Verma $\mathrm{S}$, et al. Targeting cardiovascular inflammation: next steps in clinical translation. Eur Heart J. 2020. https://doi.org/10.1093/ eurheartj/ehaa099.
48. Verma S, Leiter LA, Bhatt DL. CANTOS ushers in a new calculus of inflammasome targeting for vascular protection-and maybe more. Cell Metab. 2017;26(5):703-5. https://doi.org/10.1016/j. cmet.2017.09.022.

49. Bäck M, Yurdagul A Jr, Tabas I, Öörni K, Kovanen PT. Inflammation and its resolution in atherosclerosis: mediators and therapeutic opportunities. Nat Rev Cardiol. 2019;16(7):389-406. https://doi.org/10.1038/s41569-019-0169-2.

50. Mozaffarian D, Wu JH. Omega-3 fatty acids and cardiovascular disease: effects on risk factors, molecular pathways, and clinical events. J Am Coll Cardiol. 2011;58(20):2047-67. https://doi.org/ 10.1016/j.jacc.2011.06.063.

51. Bhatt DL. Eicosapentaenoic acid levels in REDUCE-IT and cardiovascular outcomes. American College of Cardiology Virtual Annual Scientific Session Together With World Congress of Cardiology (ACC 2020/WCC). 2020.

52. Mason RP, Jacob RF, Shrivastava S, Sherratt SCR, Chattopadhyay A. Eicosapentaenoic acid reduces membrane fluidity, inhibits cholesterol domain formation, and normalizes bilayer width in atherosclerotic-like model membranes. Biochim Biophys Acta. 2016;1858(12):3131-40. https://doi.org/10.1016/j.bbamem.2016. 10.002.

53.• Mason RP. New insights into mechanisms of action for omega-3 fatty acids in atherothrombotic cardiovascular disease. Curr Atheroscler Rep. 2019;21(1):2. https://doi.org/10.1007/s11883019-0762-1. In this review, the author examined possible mechanisms by which EPA exerts its powerful reduction in $\mathrm{CV}$ risk that was greater than expected given the degree of triglyceride lowering.

54. Mason RP, Jacob RF. Eicosapentaenoic acid inhibits glucoseinduced membrane cholesterol crystalline domain formation through a potent antioxidant mechanism. Biochim Biophys Acta. 2015;1848(2):502-9. https://doi.org/10.1016/j.bbamem.2014.10. 016.

55. Sherratt SCR, Juliano RA, Mason RP. Eicosapentaenoic acid (EPA) has optimal chain length and degree of unsaturation to inhibit oxidation of small dense LDL and membrane cholesterol domains as compared to related fatty acids in vitro. Biochim Biophys Acta Biomembr. 1862;2020(7):183254. https://doi.org/10.1016/j. bbamem.2020.183254.

56. Bays HE, Ballantyne CM, Braeckman RA, Stirtan WG, Soni PN. Icosapent ethyl, a pure ethyl ester of eicosapentaenoic acid: effects on circulating markers of inflammation from the MARINE and ANCHOR studies. Am J Cardiovasc Drugs. 2013;13(1):37-46. https://doi.org/10.1007/s40256-012-0002-3.

57. Dunbar RL, Nicholls SJ, Maki KC, Roth EM, OrloffDG, Curcio D, et al. Effects of omega-3 carboxylic acids on lipoprotein particles and other cardiovascular risk markers in high-risk statin-treated patients with residual hypertriglyceridemia: a randomized, controlled, double-blind trial. Lipids Health Dis. 2015;14:98. https:// doi.org/10.1186/s12944-015-0100-8.

$58 . \bullet$ Mason RP, Libby P, Bhatt DL. Emerging mechanisms of cardiovascular protection for the omega-3 fatty acid eicosapentaenoic acid. Arterioscler Thromb Vasc Biol. 0(0): ATVBAHA.119.313286. https://doi.org/10.1161/ATVBAHA. 119.313286. In this review, the authors looked at the differences between types of $n-3$ fatty acids, such as integration in cell membranes and effects on endothelial function, that could potentially explain the mechanisms of EPA's CV benefits.

59. Lev EI, Solodky A, Harel N, Mager A, Brosh D, Assali A, et al. Treatment of aspirin-resistant patients with omega-3 fatty acids versus aspirin dose escalation. J Am Coll Cardiol. 2010;55(2):114-21. https://doi.org/10.1016/j.jacc.2009.08.039.

60. Adili R, Hawley M, Holinstat M. Regulation of platelet function and thrombosis by omega- 3 and omega- 6 polyunsaturated fatty 
acids. Prostaglandins Other Lipid Mediat. 2018;139:10-8. https:// doi.org/10.1016/j.prostaglandins.2018.09.005.

61. Abeywardena MY, Head RJ. Longchain n-3 polyunsaturated fatty acids and blood vessel function. Cardiovasc Res. 2001;52(3):36171. https://doi.org/10.1016/s0008-6363(01)00406-0.

62. Clubb FJ, Schmitz JM, Butler MM, Buja LM, Willerson JT, Campbell WB. Effect of dietary omega-3 fatty acids on serum lipids, platelet function, and atherosclerosis in Watanabe heritable hyperlipidemic rabbits. Arteriosclerosis. 1989;9(4):529-37. https:// doi.org/10.1161/01.atv.9.4.529.

63. Adili R, Voigt EM, Bormann JL, Foss KN, Hurley LJ, Meyer ES, et al. In vivo modeling of docosahexaenoic acid and eicosapentaenoic acid-mediated inhibition of both platelet function and accumulation in arterial thrombi. Platelets. 2019;30(2):271-9. https://doi.org/10.1080/09537104.2017.1420154.

64. Gajos G, Rostoff P, Undas A, Piwowarska W. Effects of polyunsaturated omega-3 fatty acids on responsiveness to dual antiplatelet therapy in patients undergoing percutaneous coronary intervention: the OMEGA-PCI (omega-3 fatty acids after PCI to modify responsiveness to dual antiplatelet therapy) study. J Am Coll Cardiol. 2010;55(16):1671-8. https://doi.org/10.1016/j.jacc.2009. 11.080 .

65. McLennan PL. Myocardial membrane fatty acids and the antiarrhythmic actions of dietary fish oil in animal models. Lipids. 2001;36(Suppl):S111-4. https://doi.org/10.1007/s11745-0010692-x.

66. Leaf A, Kang Jing X, Xiao YF, Billman GE. Clinical prevention of sudden cardiac death by $\mathrm{n}-3$ polyunsaturated fatty acids and mechanism of prevention of arrhythmias by n-3 fish oils. Circulation. 2003;107(21):2646-52. https://doi.org/10.1161/01.CIR. 0000069566.78305 .33 .

67. Brouwer IA, Raitt MH, Dullemeijer C, Kraemer DF, Zock PL, Morris $\mathrm{C}$, et al. Effect of fish oil on ventricular tachyarrhythmia in three studies in patients with implantable cardioverter defibrillators. Eur Heart J. 2009;30(7):820-6. https://doi.org/10.1093/eurheartj/ehp003.

68. Saravanan P, Bridgewater B, West AL, O'Neill SC, Calder PC, Davidson NC. Omega-3 fatty acid supplementation does not reduce risk of atrial fibrillation after coronary artery bypass surgery: a randomized, double-blind, placebo-controlled clinical trial. Circ Arrhythm Electrophysiol. 2010;3(1):46-53. https://doi.org/10. 1161/circep.109.899633.

69. Calo L, Bianconi L, Colivicchi F, Lamberti F, Loricchio ML, de Ruvo E, et al. N-3 fatty acids for the prevention of atrial fibrillation after coronary artery bypass surgery: a randomized, controlled trial. J Am Coll Cardiol. 2005;45(10):1723-8. https://doi.org/10.1016/j. jacc.2005.02.079.

70. Kumar S, Sutherland F, Rosso R, Teh AW, Lee G, Heck PM, et al. Effects of chronic omega-3 polyunsaturated fatty acid supplementation on human atrial electrophysiology. Heart Rhythm. 2011;8(4): 562-8. https://doi.org/10.1016/j.hrthm.2010.12.017.

71. Farquharson AL, Metcalf RG, Sanders P, Stuklis R, Edwards JR, Gibson RA, et al. Effect of dietary fish oil on atrial fibrillation after cardiac surgery. Am J Cardiol. 2011;108(6):851-6. https://doi.org/ 10.1016/j.amjcard.2011.04.036.

72. Grundy SM, Stone NJ, Bailey AL, Beam C, Birtcher KK, Blumenthal RS, et al. 2018 AHA/ACC/AACVPR/AAPA/ABC/ ACPM/ADA/AGS/APHA/ASPC/NLA/PCNA Guideline on the management of blood cholesterol: a report of the American College of Cardiology/American Heart Association Task Force on Clinical Practice Guidelines. J Am Coll Cardiol. 2019;73(24): e285-350. https://doi.org/10.1016/j.jacc.2018.11.003.

73. Mach F, Baigent C, Catapano AL, Koskinas KC, Casula M, Badimon L, et al. 2019 ESC/EAS Guidelines for the management of dyslipidaemias: lipid modification to reduce cardiovascular risk. Eur Heart J. 2020;41(1):111-88. https://doi.org/10.1093/eurheartj/ehz455.

74. American Diabetes Association. Cardiovascular disease and risk management: Standards of Medical Care in Diabetes-2020. Diabetes Care. 2020;42(Suppl 1):S111-34. https://doi.org/10. 2337/dc20-S010.

75. Orringer CE, Jacobson TA, Maki KC. National Lipid Association Scientific Statement on the use of icosapent ethyl in statin-treated patients with elevated triglycerides and high or very-high ascvd risk. J Clin Lipidol. 2019;13(6):860-72. https://doi.org/10.1016/j. jacl.2019.10.014.

76. Precoma DB, Oliveira GMM, Simao AF, Dutra OP, Coelho OR, Izar MCO, et al. Updated cardiovascular prevention guideline of the Brazilian Society of Cardiology-2019. Arq Bras Cardiol. 2019;113(4):787-891. https://doi.org/10.5935/abc.20190204.

77.• Arnold SV, Bhatt DL, Barsness GW, Beatty AL, Deedwania PC, Inzucchi SE, et al. Clinical management of stable coronary artery disease in patients with type 2 diabetes mellitus: a scientific statement from the American Heart Association. Circulation. 2020;141: e000. https://doi.org/10.1161/cir.0000000000000766. In this AHA scientific statement, the authors reviewed effective therapy in management of stable CAD in patients with diabetes, including the use of icosapent ethyl.

78. Institute for Clinical and Economic Review. ICER publishes evidence report on additive cardiovascular disease therapies. 2019. https://icer-review.org/announcements/icer-publishes-evidencereport-on-additive-cardiovascular-disease-therapies/. Accessed 07 April 20202020.

79. Weintraub WS. Cost-effectiveness of icosapent ethyl in REDUCEIT. American Heart Association Annual Scientific Session. 2019, Philadelphia, PA.

80. American College of Cardiology. REDUCE-IT analysis shows benefits of icosapent ethyl in us cohort. 2019. https://www.acc. org/latest-in-cardiology/articles/2019/11/14/13/36/sat-715am-costeffectiveness-icosapent-ethyl-reduce-it-aha-2019. Accessed 30 March 2020.

81. U.S. Food and Drug Administration. FDA approves use of drug to reduce risk of cardiovascular events in certain adult patient groups. 2019. https://www.fda.gov/news-events/press-announcements/fdaapproves-use-drug-reduce-risk-cardiovascular-events-certainadult-patient-groups. Accessed 28 March 2020.

82. Reduction of cardiovascular risk with icosapent ethyl (Vascepa). Med Lett Drugs Ther. 2020;62(1591):17-8.

83. House of Representatives. Department of Labor, Health and Human Services, and Education, and Related Agencies Appropriations Bill, 2020, Report of the Commiittee on Appropriations. 2019.

Publisher's Note Springer Nature remains neutral with regard to jurisdictional claims in published maps and institutional affiliations. 